\title{
Abnormal metabolic brain networks in Tourette syndrome
}
M. Pourfar, MD*
A. Feigin, $\mathrm{MD}^{*}$
C.C. Tang, MD, PhD
M. Carbon-Correll, MD
M. Bussa, MPH
C. Budman, MD
V. Dhawan, $\mathrm{PhD}$
D. Eidelberg, MD

Address correspondence and reprint requests to Dr. Andrew Feigin, Center for Neurosciences, The Feinstein Institute for Medical Research, 350 Community Drive, Manhasset, NY 11030

afeigin@nshs.edu

\section{ABSTRACT}

Objectives: To identify metabolic brain networks that are associated with Tourette syndrome (TS) and comorbid obsessive-compulsive disorder (OCD).

Methods: We utilized $\left[{ }^{18} \mathrm{~F}\right]$-fluorodeoxyglucose and PET imaging to examine brain metabolism in 12 unmedicated patients with TS and 12 age-matched controls. We utilized a spatial covariance analysis to identify 2 disease-related metabolic brain networks, one associated with TS in general (distinguishing TS subjects from controls), and another correlating with OCD severity (within the TS group alone).

Results: Analysis of the combined group of patients with TS and healthy subjects revealed an abnormal spatial covariance pattern that completely separated patients from controls ( $p<$ 0.0001). This TS-related pattern (TSRP) was characterized by reduced resting metabolic activity of the striatum and orbitofrontal cortex associated with relative increases in premotor cortex and cerebellum. Analysis of the TS cohort alone revealed the presence of a second metabolic pattern that correlated with OCD in these patients. This OCD-related pattern (OCDRP) was characterized by reduced activity of the anterior cingulate and dorsolateral prefrontal cortical regions associated with relative increases in primary motor cortex and precuneus. Subject expression of OCDRP correlated with the severity of this symptom $(r=0.79, p<0.005)$.

Conclusion: These findings suggest that the different clinical manifestations of TS are associated with the expression of 2 distinct abnormal metabolic brain networks. These, and potentially other disease-related spatial covariance patterns, may prove useful as biomarkers for assessing responses to new therapies for TS and related comorbidities. Neurology ${ }^{\circledR}$ 2011;76:944-952

\section{GLOSSARY}

BA = Brodmann area; DLPFC = dorsolateral prefrontal cortex; DSM-IV = Diagnostic and Statistical Manual of Mental Disorders, 4th edition; FDG $=\left[{ }^{18} \mathrm{~F}\right]$-fluorodeoxyglucose; $\mathbf{O C D}=$ obsessive-compulsive disorder; OCDRP $=$ obsessivecompulsive disorder-related pattern; $\mathbf{P C}=$ principal component; $\mathbf{P C A}=$ principal component analysis; $\mathbf{T S}=$ Tourette syndrome; TSRP = Tourette syndrome-related pattern; VOI = volume of interest; YBOCS = Yale-Brown Obsessive Compulsive Scale; YGTSS = Yale Global Tics Severity Scale.

Tourette syndrome (TS) is characterized by the presence of chronic motor and vocal tics that develop before the age of $18 .{ }^{1}$ Comorbid behavioral abnormalities are common in TS, most notably obsessive-compulsive disorder (OCD) and attention-deficit/hyperactivity disorder. OCD occurs in $50 \%-70 \%$ of patients with TS, and has been linked to TS in genetic studies. ${ }^{2}$ The neurophysiology underlying TS remains poorly understood, with varying and inconsistent neuropathologic and neuroimaging findings, attributable perhaps to the clinical heterogeneity of the disorder. ${ }^{3,4}$

Many common symptoms of TS can be viewed as a failure of neural mechanisms to suppress unwanted or abnormal behaviors, including obsessions, compulsions, hyperactivity, impulsivity, aggressivity and rage, and others. ${ }^{5,6}$ Indeed, numerous studies have implicated abnormal

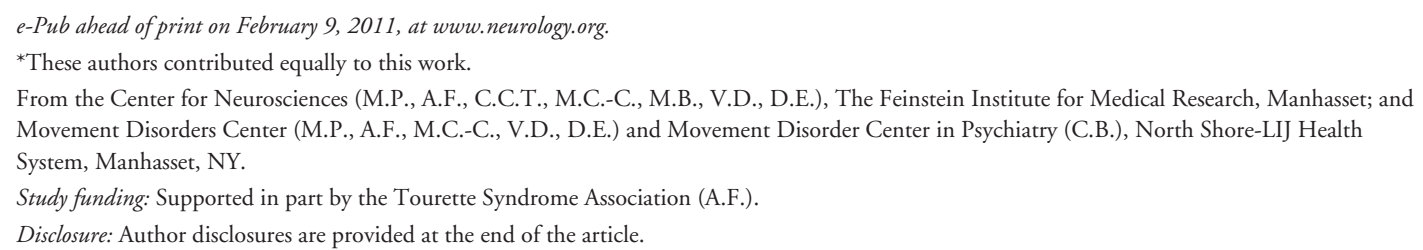


control of frontal and limbic cortico-striatothalamocortical circuits in the pathogenesis of these symptoms. ${ }^{7-9}$ Moreover, identifying single brain regions that account for the manifestations of TS is challenging, ${ }^{10}$ given that the symptoms of this condition are likely to emerge from abnormal activity of one or more spatially distributed brain networks.

In an earlier PET study, ${ }^{11}$ we identified a TS-related metabolic covariance pattern that correlated with a global measure of disability, but not with any of the specific symptoms of TS (e.g., tics, OCD). In the present study, we studied an entirely new cohort of subjects with TS to further elucidate the brain networks underlying TS through the use of a newer voxel-based network mapping approach. ${ }^{12}$ In addition, by studying subjects with TS with and without significant OCD, we were able to identify a second brain network associated with this feature of TS.

METHODS Subjects. Twelve adult subjects with TS (11 male; mean \pm SD age, $32.6 \pm 10.7$ years $)$ and 12 age-matched healthy control subjects $(5$ male; mean $\pm \mathrm{SD}$, age $32.9 \pm 7.2$ years) participated in the study. TS diagnosis was based on $D S M-I V$ diagnostic criteria. ${ }^{1}$ Subject characteristics are described in table 1. All subjects with TS were off TS-related medications

\begin{tabular}{|lllll|}
\hline Table 1 & Patient characteristics & & \\
Subject & Age, $y$ & $\begin{array}{l}\text { YGTSS total } \\
\text { tic score }\end{array}$ & $\begin{array}{l}\text { YGTSS } \\
\text { impairment score }\end{array}$ & $\begin{array}{l}\text { YBOcs } \\
\text { total score }\end{array}$ \\
\hline 1 & 26 & 21 & 25 & 0 \\
2 & 36 & 7 & 10 & 8 \\
\hline 3 & 21 & 13 & 20 & 8 \\
4 & 36 & 32 & 30 & 10 \\
\hline 5 & 23 & 11 & 20 & 10 \\
6 & 21 & 18 & 10 & 13 \\
7 & 48 & 29 & 25 & 14 \\
8 & 35 & 28 & 30 & 15 \\
9 & 20 & 26 & 40 & 16 \\
10 & 51 & 22 & 15 & 18 \\
11 & 42 & 30 & 40 & 22 \\
12 & 34 & 23 & 30 & 25 \\
\hline OCD $-{ }^{a}$ mean \pm SD & $27.2 \pm 7.1$ & $17.0 \pm 8.9^{c}$ & $19.2 \pm 8.0$ & $8.2 \pm 4.4^{\text {d }}$ \\
\hline OCD $+{ }^{b}$ mean \pm SD & $38.3 \pm 11.3$ & $26.3 \pm 3.3$ & $30.0 \pm 9.5$ & $18.3 \pm 4.3$ \\
All, mean \pm SD & $32.6 \pm 10.7$ & $21.5 \pm 8.0$ & $24.1 \pm 10.1$ & $12.2 \pm 6.7$ \\
\hline
\end{tabular}

Abbreviations: OCD = obsessive-compulsive disorder; YBOCS $=$ Yale-Brown Obsessive Compulsive Scale; YGTSS = Yale Global Tics Severity Scale.

a OCD - subgroup includes subjects 1-6.

b OCD + subgroup includes subjects 7-12.

${ }^{c} p<0.05$.

${ }^{d} p<0.005$, Student $t$ tests for comparisons between OCD - and OCD + subgroups. for $>2$ years and lacked a major Axis I diagnosis. Neurologic examinations were normal except for the presence of tics.

Clinical evaluations. Patients with TS were evaluated using the Yale Global Tics Severity Scale (YGTSS ${ }^{13,14}$ ) and the YaleBrown Obsessive Compulsive Scale (YBOCS). Six of the 12 subjects with TS were selected to have clinically significant OCD symptoms $(\mathrm{OCD}+)$ based upon a YBOCS total score of $>14$ (YBOCS score for OCD $+=18.3 \pm 4.3$; OCD $-=$ $8.2 \pm 4.4)$.

PET procedure. $\left[{ }^{18} \mathrm{~F}\right]$-Fluorodeoxyglucose (FDG) PET imaging was performed in each subject using the GE Advance tomograph (GEMS, Milwaukee, WI) at North Shore University Hospital with a reconstructed resolution of 4.5-7.0 mm (center to edge of field of view) in the transaxial direction and 6.0-7.5 $\mathrm{mm}$ in the axial direction. ${ }^{15}$ Subjects fasted overnight. They received $185-370 \mathrm{MBq}(5-10 \mathrm{mCi})$ FDG IV and were scanned for 20 minutes beginning 35 minutes after injection. Image acquisition was performed with the subject's eyes open in a dimly lit room with minimal auditory stimulation. Subjects were instructed not to suppress tics during the imaging session.

Data analysis. TS pattern identification. To identify and characterize a significant spatial covariance pattern associated with TS, patient and control scans were analyzed using a voxelbased spatial covariance approach as described elsewhere, ${ }^{12,16}$ using software available at http://www.fil.ion.ucl.ac.uk/spm/ext/ \#SSM. This fully automated routine rapidly performs principal component analysis (PCA) computation on groups of brain images transformed into a common anatomic space. To delineate topographies associated with TS, the analysis was performed on the combined group of patients and controls. After we obtained a set of linearly independent covariance patterns (i.e., principal components [PCs]), logistical regression was used to select the combination of one or more patterns for which measures of individual subject expression (subject scores) provided the best discrimination between TS subjects and controls.

The choice of patterns was based upon the following statistical criteria. 1) We limited the analysis to the first 6 PCs (typically accounting for approximately $50 \%$ of the total subject $X$ voxel variance in the analysis of FDG PET scans of this sample size). ${ }^{17,18}$ Subject scores for these patterns were entered singly and in all possible combinations into a series of logistical regression models, with the group (patients vs controls) as the dependent variable in each model. The model with the lowest Akaike Information Criterion value was selected as the one that best distinguished between the patient and control groups. ${ }^{19}$ 2) The $\chi^{2}$ statistic and $p$ value of the likelihood ratio test for the model were used to indicate the level of discrimination between subjects with TS and controls. The PCs selected for the best model were then linearly combined to yield a single spatial covariance TS-related pattern (TSRP). Subject scores for this pattern were $z$-transformed so that the mean value was 0 and the $S D$ was 1 for the control group. 3) TSRP voxel weights were tested for reliability using a bootstrap resampling procedure. ${ }^{20}$

The anatomic localization of each reported network-related region was determined using the Talairach space utility available at http://www.ihb.spb.ru/ pet_lab/TSU/TSUMain.html. To determine the degree to which local metabolic activity in the individual TSRP regions could independently differentiate patients from controls, we placed spherical volumes of interest (VOIs; radius $=5$ $\mathrm{mm}$ ) centered at each region's peak voxel using an automated algorithm (available at http://feinsteinneuroscience.org/software). Each VOI measure was ratio normalized by the global metabolic rate for 
A TS-related metabolic pattern

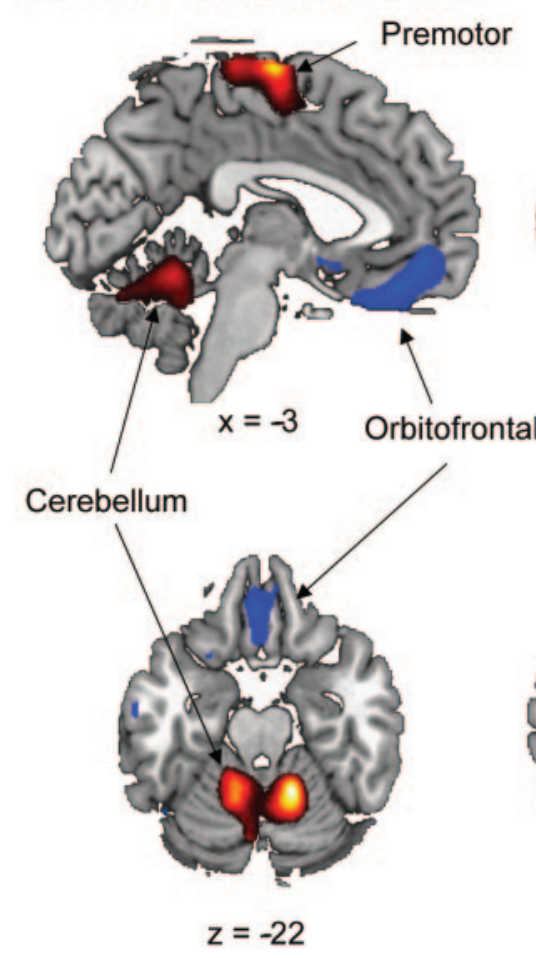

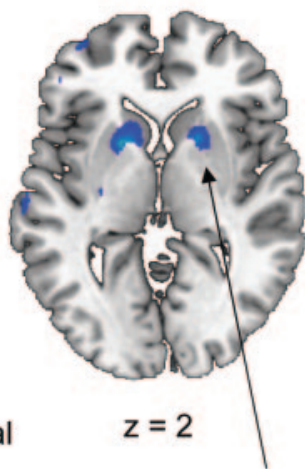

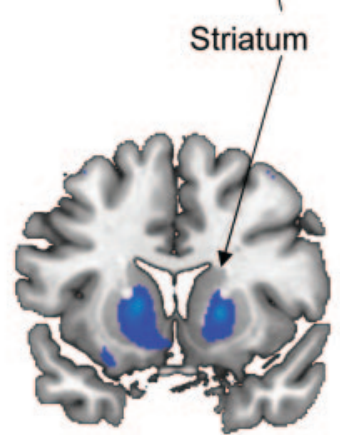

$y=12$
B

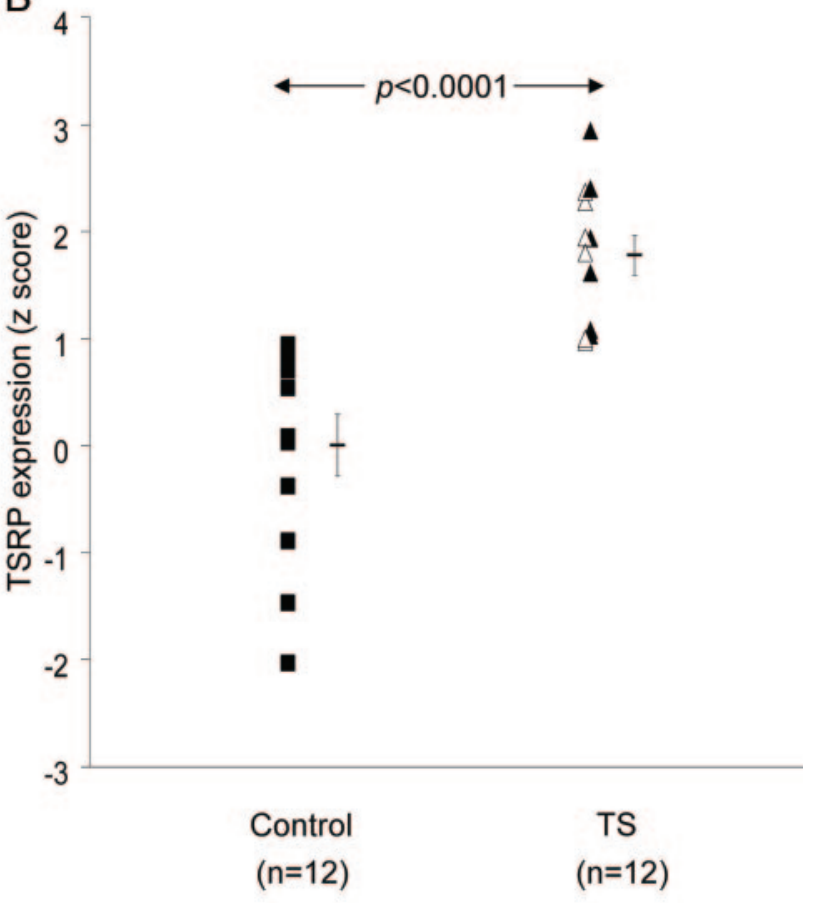

(A) This pattern, identified by spatial covariance analysis of FDG PET scans from 12 patients with TS and 12 healthy controls, was characterized by metabolic increases in bilateral premotor cortices and cerebellum covarying with metabolic decreases in caudate/putamen and orbitofrontal cortices. (B) The expression of this pattern discriminated the patients (triangles) from healthy controls (squares; $p<0.001$ ), but did not discriminate between the patients with TS with (closed triangles) and without obsessive-compulsive disorder (open triangles; $p=0.80$ ). The covariance map was overlaid on T1weighted magnetic resonance template images. The voxel weights for the pattern were thresholded at $Z=3.09(p=0.001)$ and were demonstrated to be reliable $(p<0.001)$ by bootstrap resampling (see text). Voxels with positive region weights (relative metabolic increases) are color-coded red and voxels with negative region weights (relative metabolic decreases) are color-coded blue. Error bar represents 1 SEM.

the scan. VOI values for the 2 groups were compared using 2-tailed Student $t$ tests.

OCD pattern identification. To identify and characterize a significant OCD-related pattern within the TS cohort, we employed a correlational PCA strategy, analogous to that described by us previously. ${ }^{21}$ In this exploratory analysis, PCA was applied to the patient sample alone, without including controls. The YBOCS scores of the 12 patients were normally distributed within a wide range of $0-25$. This variability allowed for the derivation of a spatial covariance OCD-related pattern (OCDRP) using a PCA-based strategy to identify metabolic networks for which subject expression correlates with individual differences in the patient group. ${ }^{17,21}$ After generating the first 6 PCs, we used multiple linear regression to correlate the expression of these patterns, singly or in linear combination, with YBOCS total ratings; the best model was selected based on a stepwise procedure analogous to that described above. The selected PCs in the best model were then used in a linear combination to yield a single OCDRP. Voxel weights for this pattern were further tested for reliability using bootstrap resampling. Using an automated voxel-based pattern quantification algorithm, ${ }^{12,22}$ OCDRP subject scores were computed for each of the 12 healthy control scans. As described above, pattern expression values for the patients and controls were $z$-transformed so that the mean value was 0 and the SD was 1 for the control group. Finally, we assessed correlations between local metabolic activity and YBOCS at each OCDRP region using VOI analysis as described above. Following global normalization, the regional measures were correlated with OCD severity values computing Pearson product-moment correlation coefficients.

Region weights for the TSRP were directly compared to those for the OCDRP using a voxel-by-voxel correlational approach (software available at www.feinsteinneuroscience.org/ software). The $R^{2}$ correlation coefficient of 2 images is calculated as the square of the Pearson correlation coefficient across all corresponding voxels with nonzero region weights on both patterns. For this calculation, images are assumed to be previously spatially normalized in 3-dimensional space and only voxels that are not 0 in both images are considered. Voxels from each image are formatted into a single vector by appending successive rows in each plane of the image. The 2 vectors are input into the MATLAB statistical routine corr to calculate the Pearson coefficient.

Standard protocol approvals, registrations, and patient consents. This study was approved by the Institutional Review Board of North Shore-LIJ Health System. Written consent was obtained from each subject following detailed explanation of the procedures.

RESULTS TS metabolic pattern. Spatial covariance analysis revealed a metabolic pattern (linear combi- 
Table 2 Brain regions with significant contributions to the Tourette syndromerelated pattern (TSRP) and to the obsessive-compulsive disorderrelated pattern (OCDRP)

\begin{tabular}{|c|c|c|c|}
\hline \multirow[b]{2}{*}{ Brain region } & \multicolumn{3}{|c|}{ Coordinates $^{a, b}$} \\
\hline & $\mathbf{x}$ & y & z \\
\hline \multicolumn{4}{|l|}{ TSRP } \\
\hline \multicolumn{4}{|l|}{ Metabolic increases } \\
\hline Premotor cortex, BA 6 & 2 & -10 & 68 \\
\hline Cerebellum, hemisphere, lobule IV-VIc , left & -14 & -46 & -25 \\
\hline Cerebellum, hemisphere, lobule IV-VI' , right & 18 & -54 & -28 \\
\hline \multicolumn{4}{|l|}{ Metabolic decreases } \\
\hline Caudate/putamen left & -18 & 8 & 2 \\
\hline Caudate/putamen right & 18 & 12 & 2 \\
\hline Orbitofrontal cortex, BA 11 & -6 & 50 & -4 \\
\hline \multicolumn{4}{|l|}{ OCDRP } \\
\hline \multicolumn{4}{|l|}{ Metabolic increases } \\
\hline Primary motor cortex, BA 4 & 16 & -26 & 54 \\
\hline Precuneus, BA 7 & 28 & -52 & 44 \\
\hline Fusiform gyrus, BA 37 & 44 & -54 & -8 \\
\hline \multicolumn{4}{|l|}{ Metabolic decreases } \\
\hline Dorsolateral prefrontal cortex, BA 9 & 36 & 16 & 30 \\
\hline Anterior cingulate, BA 32 & 16 & 36 & 8 \\
\hline
\end{tabular}

Abbreviation: $\mathrm{BA}=$ Brodmann area.

a Montreal Neurological Institute standard space. ${ }^{39}$

${ }^{b} p<0.001$ (significance of stability in peak voxel according to bootstrap estimation).

${ }^{\mathrm{c}}$ According to Atlas of Schmahmann. ${ }^{40}$

nation of PCs $1-4$, accounting for $17.7 \%$ of the total subject $X$ region variance) that discriminated the TS from the healthy subjects $\left(\chi^{2}=31.2 ; p<0.0001\right.$, likelihood ratio test). This TS-related metabolic covariance pattern (TSRP) (figure 1A) was characterized by increased activity bilaterally in premotor cortex and cerebellum, associated with relative reductions in caudate/putamen and orbitofrontal cortices. Voxel weights for this pattern (table 2, top) were stable $(p<0.001)$ by bootstrap estimation. Subject scores for this pattern (figure 1B) fully separated the patients with TS from the controls $(p<0.0001$; Student $t$ test) at a cutoff value of 0.95 . TSRP expression did not discriminate between the $\mathrm{OCD}+$ and OCD - TS subgroups ( $p=0.80$; Student $t$ test) and did not correlate with any of the clinical measures of TS symptom severity ( $p>0.12$ for YGTSS total tic score, impairment score, and YBOCS total score).

Metabolic activity measured at the major TSRP nodes (premotor cortex, cerebellum, and caudate/ putamen) differed for the TS and control groups ( $p<0.01$, Student $t$ tests) (figure 2). Regional metabolic activity in the orbitofrontal cortex did not reach significance $(p=0.09)$. These region-level group differences were less robust than the networklevel measurements; substantial overlap was present between patient and control values in the individual areas.

OCD metabolic pattern. To identify a spatial covariance pattern associated with OCD in TS, we restricted the analysis to scans from the patient cohort alone. This analysis disclosed a second spatial covariance pattern (linear combination of PCs 2 and 5, accounting for $12.0 \%$ of the subject $X$ region variance) that was unrelated to the TSRP topography ( $R^{2}=0.0025$, voxel weight correlation). This OCDrelated metabolic covariance pattern (OCDRP) (figure $3 \mathrm{~A}$ and table 2 , bottom) was characterized by reduced activity in the anterior cingulate (Brodmann area $[\mathrm{BA}] 32$ ) and dorsolateral prefrontal (BA 9) regions, associated with relative increases in the precuneus (BA 7) and primary motor cortex (BA 4). Voxel weights for the pattern were found to be stable on bootstrap estimation $(p<0.001)$.

OCDRP scores for the patients with TS correlated with YBOCS scores $(r=0.79$; $p<0.005$; figure $3 \mathrm{~B}$ ). Pattern expression was found to be higher in the OCD + relative to the OCD - patient subgroups ( $p<0.05$; Student $t$ test), although substantial overlap was present between the 2 subgroups. To further examine whether OCDRP expression was abnormally elevated in each subgroup (rather than simply relative to each other), OCDRP scores in the $\mathrm{OCD}+$ and $\mathrm{OCD}-$ subjects were compared to pattern scores computed prospectively in the healthy subjects. There was a difference across the $\mathrm{OCD}+$, OCD - , and control groups $\left(F_{2,23}=5.3, p<0.05\right.$, one-way analysis of variance). Relative to the controls, OCDRP expression was elevated in the $\mathrm{OCD}+(p<0.05)$ but not in the OCD - patient subgroups ( $p=0.55$ ) (figure $3 \mathrm{C}$ ). OCDRP expression did not correlate with tic severity or with the other clinical measures ( $p>0.48$ ). In addition, there was no correlation between TSRP and OCDRP expression in the subjects with TS $(p=0.73)$. We additionally measured local metabolic activity at the major OCDRP nodes. Regional metabolic activity correlated with YBOCS scores in the dorsolateral prefrontal cortex (DLPFC) $(r=-0.61 ; p<0.05)$, but not in the other OCDRP regions $(p>0.10)$.

DISCUSSION In the present study, we identified a metabolic pattern that distinguished subjects with TS from controls, as well as a second pattern within the TS cohort that differentiated those with from those without prominent OCD features. Both brain networks involve regions typically associated with motor activity (e.g., motor cortices) as well as those more commonly associated with behavioral changes (e.g., anterior cingulate and prefrontal cortical regions). We found that no single region within these 
Figure 2 Regional glucose metabolism measured at representative nodes of the Tourette syndrome (TS)related pattern

Caudate/Putamen (18 12 2)

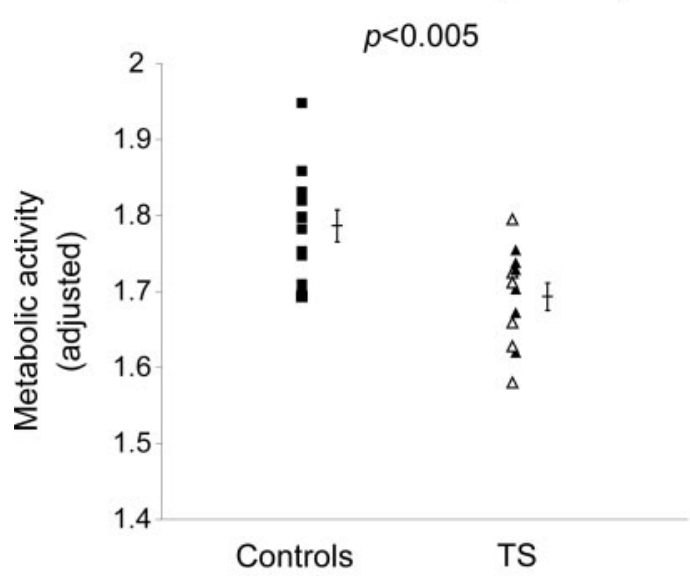

Orbitofrontal cortex (-6 50 -4)

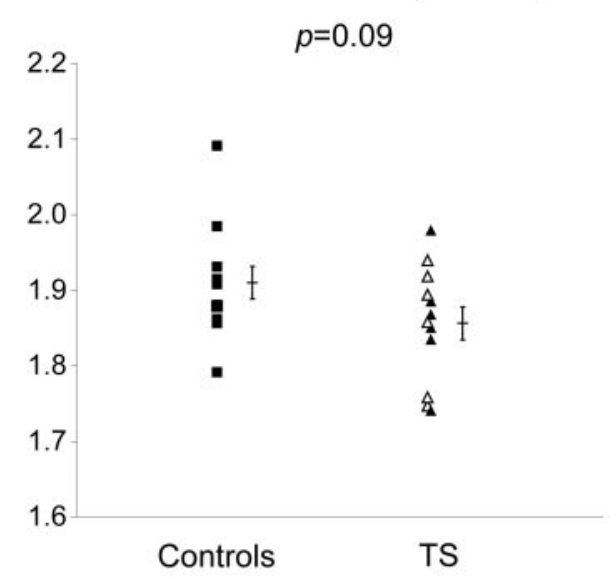

Premotor cortex (2 -10 68)
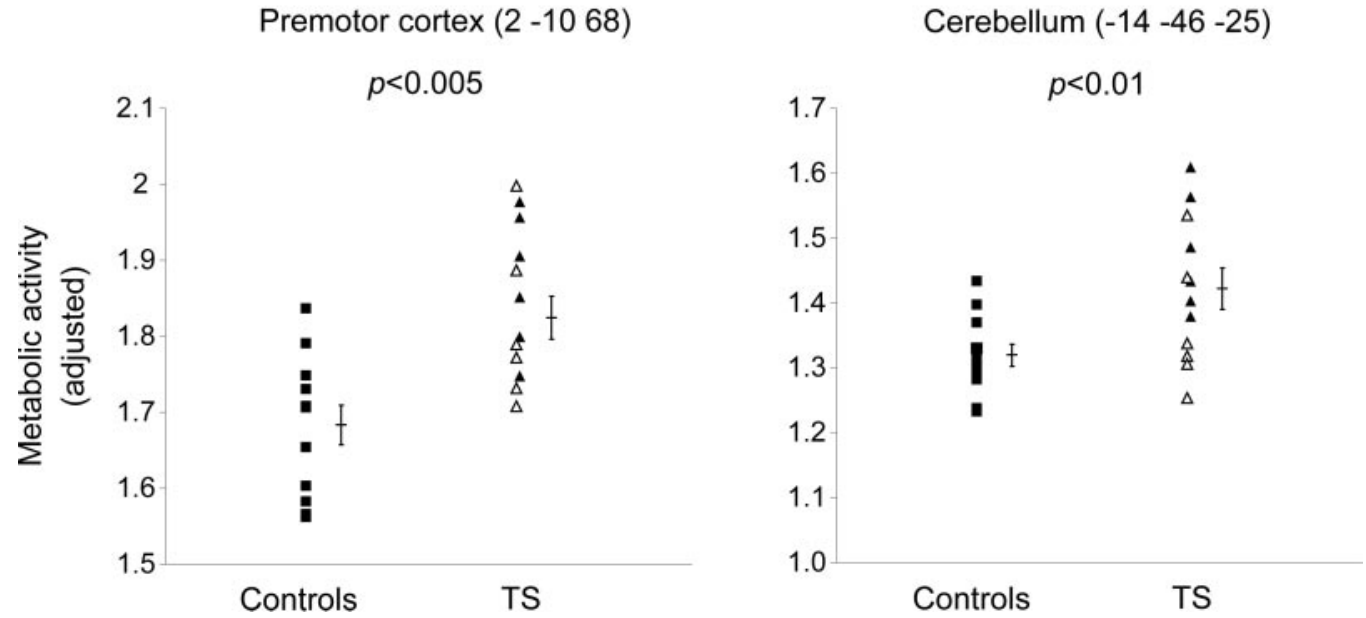

Within the pattern, normalized glucose metabolism was decreased in the caudate/putamen $(p<0.005)$ and elevated in the premotor cortex $(p<0.005)$ and cerebellum $(p<0.01)$ in the TS patient group (triangles) relative to the control group (squares). A trend of decrease in regional metabolism was also found in the patients with TS compared to controls ( $p=$ 0.09). Regional metabolism did not discriminate between the patients with TS with (closed triangles) and without OCD (open triangles) in any of the 4 regions ( $p>0.07$ ).

patterns independently correlated with TS or OCD severity, though individual subject pattern expression separated the TS group from the controls, and for the OCD pattern, correlated with OCD clinical severity. The findings support the idea that the clinical manifestations of TS and OCD emerge through the abnormal activity of specific resting-state functional brain networks. ${ }^{11}$

The TSRP was characterized by increased premotor and cerebellar metabolism covarying with decreased striatal and orbitofrontal metabolism. Other imaging-related TS studies have also found increased metabolism in premotor cortices ${ }^{23-25}$ and cerebellum, ${ }^{25}$ as well as decreased metabolism in the striatum and orbitofrontal cortex. ${ }^{23}$ The involvement of these regions may reflect the interaction between the defining core motor features of TS (i.e., tics), driven primarily by the striatum and cerebellum, and related behavioral features mediated by frontal and prefrontal involvement. The TSRP metabolic topography thus likely reflects a functional aggregate of the motor and nonmotor features of TS. In this regard, it is not surprising that pattern expression in individual subjects did not correlate with specific symptom (e.g., tics, OCD) severity.

These findings comport with our earlier study in which a region of interest-based TS network was identified that separated the TS group from controls and correlated with YGTSS global impairment, but not with specific symptoms such as tics and OCD. ${ }^{11}$ In that study, we were not able to identify other brain networks that correlated with specific symptoms. In the current study, however, to identify an OCD-related pattern, we intentionally recruited sub- 


\section{A OCD-related metabolic pattern}
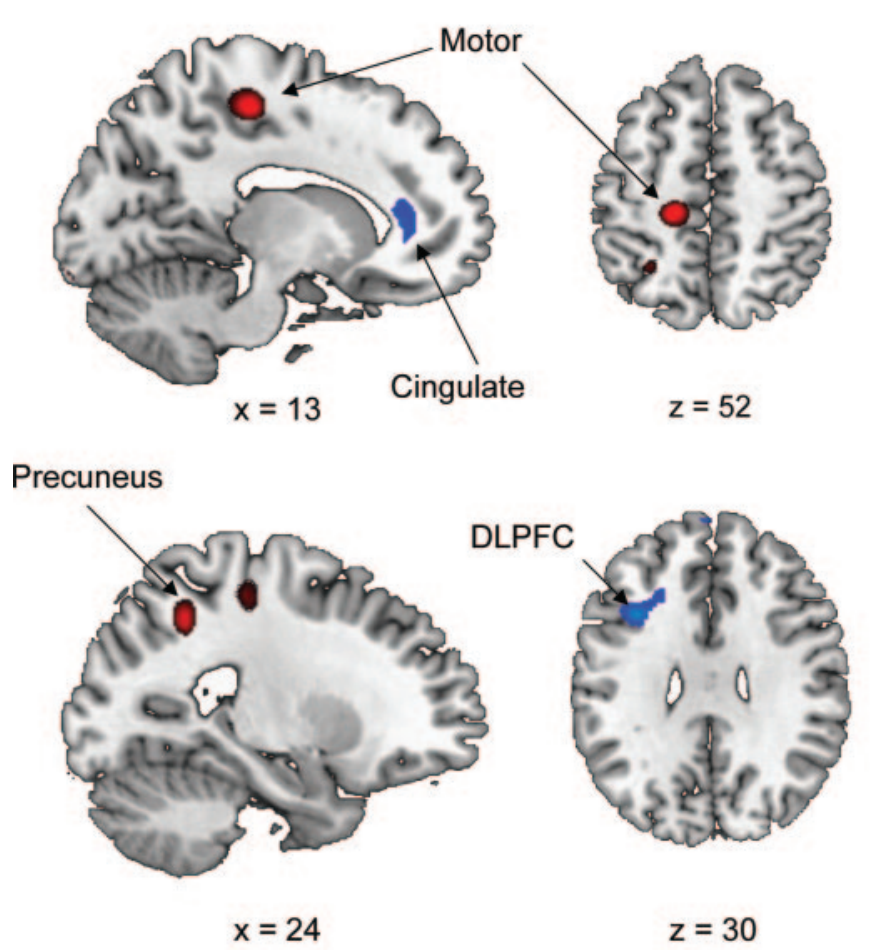
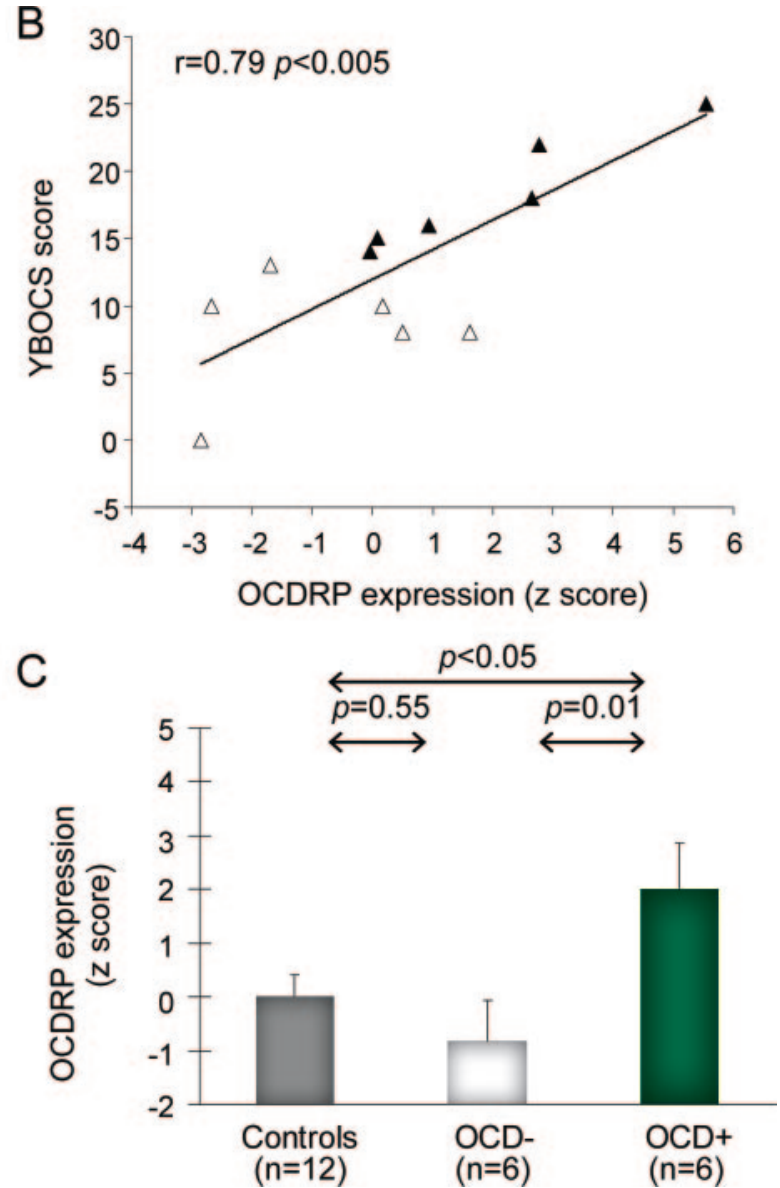

(A) This pattern, identified by spatial covariance analysis of FDG PET scans from 12 patients with Tourette syndrome (TS), was characterized by metabolic reductions in the cingulate and dorsolateral prefrontal cortex (DLPFC) covarying with relative metabolic increases in the precuneus and primary motor cortex. (B) The expression of this pattern correlated with Yale-Brown Obsessive Compulsive Scale (YBOCS) scores $(p<0.005)$ and discriminated between the patients with TS with (closed triangles) and without OCD (open triangles) ( $p<0.05$ ). (C) Relative to normal controls (gray), pattern expression was elevated in the TS OCD + subgroup (green, $p<0.05$ ), but not in the OCD - subgroup (white, $p=0.55$ ). The covariance map was overlaid on T1-weighted magnetic resonance template images. Voxel weights for the pattern were thresholded at $Z=2.33, p=0.01$, and were demonstrated to be reliable ( $p<$ 0.001 ) by bootstrap resampling (see text). Voxels with positive region weights (metabolic increases) are color-coded red and voxels with negative region weights (metabolic decreases) are color-coded blue. Error bar represents 1 SEM.

jects with TS with and without significant OCD symptoms. The variability of YBOCS scores across the subjects with TS allowed for the use of the correlational PCA approach to derive an OCDRP in a relatively small sample within the TS cohort. The OCDRP identified from this exploratory analysis was characterized by relative metabolic reductions in anterior cingulate and DLPFC, covarying with relative increases in the activity of primary motor cortex and precuneus. Individual subject expression of this pattern highly correlated with OCD severity across the whole TS cohort. Consistent with this correlation, OCDRP scores also differentiated the OCD+ subjects from the $\mathrm{OCD}-$ subjects. The brain regions involved in this covariance pattern comport with earlier OCD-related neuroimaging findings. Specifically, most OCD neuroimaging studies have reported increased functional activity in the
DLPFC, ${ }^{26-29}$ precuneus, ${ }^{27,30}$ motor and premotor cortex, ${ }^{26-28}$ and anterior cingulate cortex. ${ }^{28}$ Indeed, the current findings are consistent with the results of a recent resting state fMRI study in which children with TS exhibited abnormal functional connectivity in frontoparietal circuits, principally those involving the DLPFC and precuneus. ${ }^{7}$

There are diverse hypotheses to explain the involvement of these particular regions in OCD. The involvement of the precuneus, for example, may relate to its role in self-awareness ${ }^{31,32}$ and its involvement in the OCDRP may thus reflect the patients' efforts to resist the obsessive thoughts. ${ }^{33}$ Similarly, the anterior cingulate, which connects with the precuneus, may play a role in both attention and in actions with exclusively internal motivations, ${ }^{34}$ with overactivity leading to OCD behaviors via erroneous interpretations of internally perceived errors or con- 
flicts. The identified OCD metabolic network thus incorporates many regions normally associated with OCD with the notable exception of the caudate, which did not differ metabolically between TS + OCD and TS - OCD subjects. This could reflect the fact that the TS group on the whole already had differing caudate metabolism compared with controls, thereby potentially overshadowing the subtler within-group difference.

It has been estimated that between $40 \%$ and $60 \%$ of patients with TS have significant comorbid OCD. ${ }^{35,36}$ Whether or not the OCD in TS is fundamentally different from OCD without TS has been a matter of debate. ${ }^{35,37,38}$ It has been suggested that OCD associated with TS involves more compulsive behaviors than idiopathic OCD. ${ }^{37}$ This could partly explain the involvement of the motor cortex and striatum in the OCDRP. Finally, increased activity in primary motor cortex in OCDRP might also reflect active effort to suppress tics. In this regard, it is notable that the OCD + group had more tics than the OCD - group (table 1).

There are 2 minor caveats regarding our study design. First, the OCD + and OCD - groups were not matched for tic severity. As a practical matter, this could not be avoided as subjects with more severe TS are more likely to have more tics and OCD (though correlation between YBOCS and tic scores did not reach statistical significance $[r=0.42, p=$ $0.17]$ in our cohort). Nonetheless, it is unlikely that this impacted our results given that OCDRP expression did not correlate with tic severity in this TS cohort. Second, the TS group and controls were not matched for gender. This is also not likely to have significantly affected our results given that none of the controls (including the 5 men) overlapped with the TS group for TSRP expression. Moreover, the separation of patients and controls by the TSRP scores remained significant even after controlling for the effects of a candidate gender-related metabolic covariance pattern. Nonetheless, given that TS is more common among males than females, future studies will be required to explore further the relationship (if any) between gender and TSRP. Finally, given that this study involved a relatively small sample of adult patients with TS, other unknown factors could have impacted our results, including the possibility of distant (i.e., more than 2 years) exposure to neuroleptics, potential variations in tic severity during PET scanning, and possible unknown demographic mismatches between the TS and control groups (e.g., years of education, history of cigarette smoking).

It is notable that TSRP provided better group discrimination, and that OCDRP scores correlated bet- ter with clinical measures than did the individual brain regions that contributed to these patterns. These results support the notion that TS and OCD are not merely the manifestation of specific dysfunctional brain regions, but rather are syndromes that emerge through the abnormal activity in specific brain networks. Our spatial covariance patterns, as well as findings from a resting fMRI connectivity study, ${ }^{7}$ suggest the presence of abnormal functional interactions between the involved regions in patients with TS. Nonetheless, it is also possible that these changes reflect the underlying regional pathology of TS rather than an abnormality of functional organization at the network level. That said, a significant strength of the network measures is their ability to provide an objective and quantifiable measure of disease expression in individual subjects. Future studies will assess the expression of the TSRP and OCDRP patterns in larger patient and control cohorts, ideally including non-TS-associated OCD. Indeed, it will be critical to determine whether existing or novel therapeutic interventions can suppress the activity of these and related functional brain networks. If validated in independent populations, and found to be reproducible, these patterns may find use as adjunctive outcome measures in clinical trials.

\section{AUTHOR CONTRIBUTIONS}

Statistical analysis was conducted by Dr. Chris C. Tang.

\section{ACKNOWLEDGMENT}

The authors thank Toni Fitzpatrick (The Feinstein Institute for Medical Research) for assistance with copyediting.

\section{DISCLOSURE}

Dr. Pourfar has received funding for travel or speaker honoraria from Teva Pharmaceutical Industries Ltd. and Medtronic, Inc.; has served as a consultant for Medtronic, Inc.; and serves on the speakers' bureau of Teva Pharmaceutical Industries Ltd. Dr. Feigin serves on speakers' bureaus for Allergan, Inc. and Teva Pharmaceutical Industries Ltd.; and receives research support from the NIH (NINDS, NCRR), the Dana Foundation, the Huntington's Disease Society of America, and the Tourette Syndrome Association. Dr. Tang, Dr. Carbon-Correll, and Dr. Bussa report no disclosures. Dr. Budman has served on the speakers' bureau for Eli Lilly and Company; serves on medical advisory boards for the National Tourette Syndrome Association and the Long Island TSA; and receives research support from Boehringer Ingelheim and NIH/NINDS. Dr. Dhawan receives research support from Neurologix, Inc., NIH (NIDCD, NIAID), and the Dana Foundation. Dr. Eidelberg serves on scientific advisory boards for the Thomas Hartman Foundation for Parkinson's Research, Inc., the Michael J. Fox Foundation, and The Bachmann-Strauss Dystonia and Parkinson Foundation; served as an Associate Editor for Molecular Imaging and Biology and currently serves on the editorial board of Annals of Neurology; is coinventor of patents re: Methods for using pet measured metabolism to determine cognitive impairment; serves as a consultant for Neurologix, Inc.; receives research support from NIH (NINDS, NCRR, NIDCD, NIAID), the High Q Foundation, the Dana Foundation, and the Bachmann-Strauss Dystonia and Parkinson Foundation; and has served as a consultant in medico-legal cases.

Received April 12, 2010. Accepted in final form September 27, 2010. 


\section{REFERENCES}

1. American Psychiatric Association. Diagnosis and Statistical Manual of Mental Disorders, 4th ed. Washington, DC: American Psychiatric Association; 1994.

2. Pauls DL, Leckman JF. The inheritance of Gilles de la Tourette's syndrome and associated behaviors: evidence for autosomal dominant transmission. N Engl J Med 1986;315:993-997.

3. Albin RL, Mink JW. Recent advances in Tourette syndrome research. Trends Neurosci 2006;29:175-182.

4. Swerdlow NR. Tourette syndrome: current controversies and the battlefield landscape. Curr Neurol Neurosci Rep 2005;5:329-331.

5. Albin RL. Neurobiology of basal ganglia and Tourette syndrome: striatal and dopamine function. Adv Neurol 2006; 99:99-106.

6. Swain JE, Scahill L, Lombroso PJ, King RA, Leckman JF. Tourette syndrome and tic disorders: a decade of progress. J Am Acad Child Adolesc Psychiatry 2007;46:947-968.

7. Church JA, Fair DA, Dosenbach NU, et al. Control networks in paediatric Tourette syndrome show immature and anomalous patterns of functional connectivity. Brain 2009; 132:225-238.

8. Makki MI, Govindan RM, Wilson BJ, Behen ME, Chugani HT. Altered fronto-striato-thalamic connectivity in children with Tourette syndrome assessed with diffusion tensor MRI and probabilistic fiber tracking. J Child Neurol 2009;24:669-678.

9. Mazzone L, Yu S, Blair C, et al. An FMRI study of frontostriatal circuits during the inhibition of eye blinking in persons with Tourette syndrome. Am J Psychiatry 2010; 167:341-349.

10. Roessner V, Overlack S, Baudewig J, Dechent P, Rothenberger A, Helms G. No brain structure abnormalities in boys with Tourette's syndrome: a voxel-based morphometry study. Mov Disord 2009;24:2398-2403.

11. Eidelberg D, Moeller JR, Antonini A, et al. The metabolic anatomy of Tourette's syndrome. Neurology 1997;48: 927-934.

12. Eidelberg D. Metabolic brain networks in neurodegenerative disorders: a functional imaging approach. Trends Neurosci 2009;32:548-557.

13. Storch EA, Murphy TK, Fernandez M, et al. Factoranalytic study of the Yale Global Tic Severity Scale. Psychiatry Res 2007;149:231-237.

14. Storch EA, Murphy TK, Geffken GR, et al. Reliability and validity of the Yale Global Tic Severity Scale. Psychol Assess 2005;17:486-491.

15. Huang C, Mattis P, Perrine K, Brown N, Dhawan V, Eidelberg D. Metabolic abnormalities associated with mild cognitive impairment in Parkinson disease. Neurology 2008;70:1470-1477.

16. Spetsieris PG, Ma Y, Dhawan V, Eidelberg D. Differential diagnosis of parkinsonian syndromes using PCA-based functional imaging features. Neuroimage 2009; 45:12411252.

17. Moeller JR, Ishikawa T, Dhawan V, et al. The metabolic topography of normal aging. J Cereb Blood Flow Metab 1996;16:385-398.

18. Eckert T, Tang C, Ma Y, et al. Abnormal metabolic networks in atypical parkinsonism. Mov Disord 2008;23: $727-733$.
19. Habeck C, Foster NL, Perneczky R, et al. Multivariate and univariate neuroimaging biomarkers of Alzheimer's disease. Neuroimage 2008;40:1503-1515.

20. Efron B, Tibshirani R. An Introduction To The Bootstrap. New York: CRC Press; 1994.

21. Huang C, Mattis P, Tang C, Perrine K, Carbon M, Eidelberg D. Metabolic brain networks associated with cognitive function in Parkinson's disease. Neuroimage 2007;34: $714-723$.

22. Ma Y, Tang C, Spetsieris PG, Dhawan V, Eidelberg D. Abnormal metabolic network activity in Parkinson's disease: test-retest reproducibility. J Cereb Blood Flow Metab 2007;27:597-605.

23. Braun AR, Stoetter B, Randolph C, et al. The functional neuroanatomy of Tourette's syndrome: an FDG-PET study: I: regional changes in cerebral glucose metabolism differentiating patients and controls. Neuropsychopharmacology 1993;9:277-291.

24. Stern E, Silbersweig DA, Chee KY, et al. A functional neuroanatomy of tics in Tourette syndrome. Arch Gen Psychiatry 2000;57:741-748.

25. Lerner A, Bagic A, Boudreau EA, et al. Neuroimaging of neuronal circuits involved in tic generation in patients with Tourette syndrome. Neurology 2007;68:1979-1987.

26. Breiter HC, Rauch SL. Functional MRI and the study of OCD: from symptom provocation to cognitive-behavioral probes of cortico-striatal systems and the amygdala. Neuroimage 1996;4:S127-S138.

27. Rauch SL, Jenike MA, Alpert NM, et al. Regional cerebral blood flow measured during symptom provocation in obsessive-compulsive disorder using oxygen 15-labeled carbon dioxide and positron emission tomography. Arch Gen Psychiatry 1994;51:62-70.

28. Mataix-Cols D, Wooderson S, Lawrence N, Brammer MJ, Speckens A, Phillips ML. Distinct neural correlates of washing, checking, and hoarding symptom dimensions in obsessive-compulsive disorder. Arch Gen Psychiatry 2004; 61:564-576.

29. Schienle A, Schafer A, Stark R, Walter B, Vaitl D. Neural responses of OCD patients towards disorder-relevant, generally disgust-inducing and fear-inducing pictures. Int J Psychophysiol 2005;57:69-77.

30. Nakao T, Nakagawa A, Yoshiura T, et al. Brain activation of patients with obsessive-compulsive disorder during neuropsychological and symptom provocation tasks before and after symptom improvement: a functional magnetic resonance imaging study. Biol Psychiatry 2005;57:901910 .

31. Cavanna AE, Trimble MR. The precuneus: a review of its functional anatomy and behavioural correlates. Brain 2006;129:564-583.

32. Gusnard DA, Akbudak E, Shulman GL, Raichle ME. Medial prefrontal cortex and self-referential mental activity: relation to a default mode of brain function. Proc Natl Acad Sci USA 2001;98:4259-4264.

33. Rotge JY, Guehl D, Dilharreguy B, et al. Provocation of obsessive-compulsive symptoms: a quantitative voxelbased meta-analysis of functional neuroimaging studies. J Psychiatry Neurosci 2008;33:405-412.

34. Rapoport JL, Wise SP. Obsessive-compulsive disorder: evidence for basal ganglia dysfunction. Psychopharmacol Bull 1988;24:380-384.

35. Anholt GE, Cath DC, Emmelkamp PM, van Oppen P, Smit JH, van Balkom AJ. Do obsessional beliefs discrimi- 
nate OCD without tic patients from OCD with tic and Tourette's syndrome patients? Behav Res Ther 2006;44: 1537-1543.

36. Kano Y, Ohta M, Nagai Y, Scahill L. Association between Tourette syndrome and comorbidities in Japan. Brain Dev 2010;32:201-207.

37. Jaisoorya TS, Reddy YC, Srinath S, Thennarasu K. Obsessive-compulsive disorder with and without tic disorder: a comparative study from India. CNS Spectr 2008;13: 705-711.
38. Como PG, LaMarsh J, O’Brien KA. Obsessive-compulsive disorder in Tourette's syndrome. Adv Neurol 2005;96: $249-261$.

39. Collins DL, Neelin P, Peters TM, Evans AC. Automatic $3 \mathrm{D}$ intersubject registration of $\mathrm{MR}$ volumetric data in standardized Talairach space. J Comput Assist Tomogr 1994;18:192-205.

40. Schmahmann JD, Doyon J, Toga AW, Petrides M, Evans AC. MRI Atlas of the Human Cerebellum. San Diego: Academic Press; 2000.

\section{Visit the Neurology ${ }^{\circledR}$ Web Site at www.neurology.org}

- Enhanced navigation format

- Increased search capability

- Highlighted articles

- Detailed podcast descriptions

- RSS Feeds of current issue and podcasts

- Personal folders for articles and searches

- Mobile device download link

- AAN Web page links

- Links to Neurology Now ${ }^{\circledR}$, Neurology Today ${ }^{\circledR}$, and Continuum ${ }^{\circledR}$

- Resident \& Fellow subsite

A

twitter Follow Neurolog $y^{\circledR}$ on Twitter: http://twitter.com/GreenJournal 